\title{
ATUAÇÃO DA LIGA ACADÊMICA NA PREVENÇÃO DE INCAPACIDADES EM HANSENÍASE
}

\author{
É. N. Sousa'; M. A. M. Fernandes²; A. K. Q. Silva ${ }^{3}$; P. N. Silva ${ }^{4}$; C. M. G. D. Florêncio ${ }^{5}$; \& P. S. F. Nogueira ${ }^{6}$.
}

${ }^{1}$ Graduanda em Enfermagem pela Universidade Federal do Ceará (UFC). Bolsista IC/CNPq/UFC. Integrante da Liga Acadêmica em Doenças Estigmatizantes (LADES). E-mail: ericanascimento.ufc@ gmail.com; ${ }^{2}$ Graduanda em Enfermagem pela UFC. Bolsista IC/UFC. Integrante da LADES. E-mail: psychosocialamanda@ gmail.com; ${ }^{3}$ Graduanda em Enfermagem pela UFC. Bolsista PREX/UFC. Integrante LADES. E-mail: kalinekeiroz@ gmail.com; ${ }^{4}$ Graduanda em Enfermagem pela UFC. Bolsista PAIP/UFC. E-mail: paatricia12silva@ gmail.com; ${ }^{5}$ Doutora em Saúde Coletiva e docente do Departamento de Saúde Comunitária da UFC. Integrante LADES E-mail: caroline.florencio1985@ gmail.com; ${ }^{6}$ Doutora Professora do Departamento de Enfermagem da Universidade Federal do Ceará (UFC) e Coordenadora da LADES. E-mail: sachanogueiraufc@gmail.com.

Artigo submetido em setembro/2018

\section{RESUMO}

A hanseníase é uma doença infecciosa crônica que pode causar incapacidades permanentes quando diagnosticada tardiamente. Objetivou-se relatar atividades extensionistas na prevenção de incapacidades em pacientes acometidos pela hanseníase. Trata-se de um estudo descritivo das atividades realizadas no Ambulatório de Dermatologia do Hospital Universitário Walter Cantídio, campo de atuação da Liga Acadêmica em Doenças Estigmatizantes (LADES) da Universidade Federal do Ceará. Durante as consultas os principais acometimentos encontrados foram: dores neuropáticas, espessamento de nervos periféricos, ressecamento e descamação da pele, ressecamento de nariz e olhos, mal perfurante plantar, alteração de sensibilidade palmar e plantar, diminuição da força muscular da mão, amiotrofia em mão e garra rígida e móvel. Foram fornecidas orientações de autocuidado, também foram feitos encaminhamentos para consultas com especialistas quando observada a necessidade. As atividades citadas proporcionam engajamento acadêmico na melhoria da qualidade de vida de pacientes com hanseníase.

PALAVRAS-CHAVE: Hanseníase; Prevenção \& Controle; Avaliação em Enfermagem.

\section{ACADEMIC GROUP IN THE PREVENTION OF LEPROSY DISABILITIES}

\begin{abstract}
Leprosy is a chronic infectious disease that can cause permanent disability when diagnosed late. The objective was to report extension activities in the prevention of disabilities in patients affected by leprosy. This is a descriptive study of the activities performed at the Dermatology Outpatient Clinic of the Walter Cantídio University Hospital, a field of action of the Academic League in Stigmatizing Diseases (LADES) of the Federal University of Ceará. During consultations, the main findings were: neuropathic
\end{abstract}

pain, thickening of peripheral nerves, dryness and scaling of the skin, dry nose and eyes, poor perforation of the plantar, alteration of palmar and plantar sensitivity, decreased hand muscle strength, amiotrophy in hand and rigid and movable claw. Selfcare guidelines were provided, referrals were also made to specialists when necessary. These activities provide academic engagement in improving the quality of life of patients with leprosy.

KEYWORDS: Leprosy; Prevention \& Control; Nursing Assessment.

\section{INTRODUÇÃO}

A hanseníase é uma doença infecciosa crônica de evolução lenta e progressiva, 
causada pelo Mycobacterium Leprae através do contato direto. O potencial incapacitante da hanseníase se dá pelo acometimento dos nervos periféricos, sua manifestação principal é através de sintomas dermatoneurológicos como lesões na pele e nervos periféricos de olhos, membros superiores e inferiores (ARAÚJO, et al., 2014).

Apesar de todos os esforços realizados pela Organização Mundial de Saúde (OMS) para o controle e eliminação da doença, é alta a prevalência da hanseníase em todo o mundo, em que houveram 214.783 casos novos por 10.000 habitantes em 2016. No Brasil a hanseníase é um grave problema de saúde pública, por seu potencial incapacitante e estigma social. Mundialmente, o país se encontra entre os três países que englobam $86 \%$ de todos os casos de hanseníase (FLORÊNCIO, 2018). No Ceará, foram notificados 21.335 casos novos da doença, no período de 2007 a 2016, sendo 6.930 na capital (CEARÁ, 2017).

A hanseníase está classificada para fins terapêuticos como Paucibacilar (PB) e Multibacilar (MB), mas assume formas clínicas diversas conforme sua manifestação em pele e nervos, sendo a resposta imunológica do paciente determinante para sua classificação em: forma inicial ou indeterminada; pólo tuberculóide (TT) (com maior resistência imunológica ao bacilo), pólo lepromatoso (LL), e formas intermediárias (borderline) (sem resistência ao bacilo) (BRASIL, 2017).

A doença evolui inicialmente com alterações da sensibilidade térmica: hiperestesia, seguidas de hipoestesia, e depois anestesia. Em seguida, ocorre perda progressiva da sensibilidade dolorosa e, por último, da tátil. Em estágios mais avançados, ocorre o comprometimento neural (ARAÚJO, et al., 2014).

Alterações neurais são comuns a todos os subtipos de hanseníase. As neurites não diagnosticadas e não tratadas são determinantes para ocorrência de incapacidades (FLORÊNCIO, 2018).

Quando as complicações hansênicas são identificadas precocemente, antes da presença de danos neurais graves, a instalação de incapacidades pode ser evitada. Nesse sentido, ações de educação em saúde para identificar novos casos de hanseníase precocemente, tratamento imediato, manejo adequado da doença, e busca ativa de contatos, são primordiais.

Este estudo justifica-se pela necessidade de compartilhar experiências acadêmicas enriquecedoras no combate à hanseníase e as suas complicações incapacitantes cada vez mais presente nos pacientes com diagnóstico tardio. Além de contribuir para o crescimento da Enfermagem enquanto ciência, motivando outros estudos nessa área. 
Assim, temos como objetivo deste estudo relatar atividades extensionistas na prevenção de incapacidades em pacientes acometidos pela hanseníase.

\section{FUNCIONALIDADE NA HANSENÍASE}

Incapacidade é toda alteração anatômica ou fisiológica num indivíduo, que o impeça ou dificulte, total ou parcialmente, permanente ou temporariamente, de desempenhar atividade e/ou convivência social normais (GONÇALVES, 1973, apud ALVES, et al. 2010).

Um importante indicador epidemiológico mundial da hanseníase é o Grau de Incapacidade Física (GIF) que deve ser testado, no mínimo, no momento do diagnóstico e após a alta. O teste compreende a avaliação de força muscular e de sensibilidade dos olhos, mãos e pés, graduado de 0 a 2 , sendo o grau zero a ausência de incapacidade por hanseníase e os graus 1 e 2 indicam alterações sensitivas/motoras de gravidade crescente (BRASIL, 2017).

O GIF é sensível para a detecção das regiões em que o diagnóstico da hanseníase está ocorrendo de forma eficiente, antes do acometimento de incapacidades, bem como demonstra regiões em que há necessidade de maiores iniciativas de busca, estratégias e investimentos no enfrentamento da hanseníase. Ele também é um indicador indireto do nível de conscientização dos sinais e sintomas da doença, do acesso aos serviços de saúde, bem como da habilidade dos profissionais da atenção primária no diagnóstico da hanseníase (OMS, 2016).

Entre as metas globais a serem alcanças até 2020 sugeridas pela Organização Mundial da Saúde, alcançar a taxa de apenas um caso com GIF 2 por um milhão de habitantes é uma das principais (OMS, 2016).

Florêncio (2018) analisando os casos notificados de hanseníase de 2001 a 2015 no Sistema de Informação de Agravos de Notificação (SINAN) encontrou um total de 612.850 casos, dos quais 6\% (36.821 casos) apresentavam GI 2, enquanto 9,06\% não foram se quer avaliados quanto ao GIF.

A consulta de Enfermagem é regulamentada por lei (Lei $N^{o} 7.498 / 86$ e Decreto $N^{\circ}$ 94.406/87) e legitimada como uma modalidade de prestação de assistência direta ao cliente. É um momento de encontro entre profissional e cliente, constituindo-se em um espaço de escuta que oportuniza a detecção de carência de cuidados não somente de aspectos físicos, mas também psicossociais, econômicos, afetivos e culturais, nesse sentido é um ambiente propício para desenvolver cuidados e prevenir incapacidades por hanseníase (DUARTE, 2009). 


\section{MATERIAIS E MÉTODOS}

Estudo descritivo de abordagem qualitativa que aborda as atividades de extensão em consultas de Enfermagem realizadas no Ambulatório de Dermatologia do Hospital Universitário Walter Cantídio (HUWC) por integrantes da Liga Acadêmica em Doenças Estigmatizantes (LADES) da Universidade Federal do Ceará (UFC). As atividades realizadas ocorrem em 16 horas semanais e o público alvo são os pacientes cadastrados no ambulatório para tratamento da hanseníase até a alta.

O Ambulatório de Dermatologia é um centro especializado no tratamento de doenças de pele entre as quais está a hanseníase. Os pacientes acompanhados no serviço recebem atendimento integral com direito a interconsultas com profissionais de outras especialidades, quando apresentam necessidade, entre as quais: ortopedia, traumatologia, oftalmologia, odontologia, estomaterapia, dentre outras. O ambulatório é referência no diagnóstico da hanseníase contando com laboratório próprio para análise de baciloscopia de esfregaço intradérmico e biópsia de pele.

Os pacientes acompanhados são oriundos da capital e região metropolitana de Fortaleza, Ceará, e têm acesso ao serviço através de encaminhamentos realizados pela atenção primária.

A LADES é um projeto de extensão do Departamento de Enfermagem da Universidade Federal do Ceará (UFC), fundada em 19 de junho de 2015, com o objetivo de contribuir para o controle da hanseníase e tuberculose no estado do Ceará, e incrementar a formação do acadêmico da área de saúde e afins, aperfeiçoando suas habilidades, atitudes, com foco na formação científica, humanística e ética. Atualmente, a LADES é formada por 16 acadêmicos do curso de Enfermagem, 1 do curso de Fisioterapia, 1 do curso de Odontologia, 1 do curso de Farmácia, 3 enfermeiros, e 2 docentes. A LADES também realiza atividades itinerantes voltadas a comunidade em espaços públicos, onde o foco é a orientação e avaliação sobre hanseníase e tuberculose.

\section{RESULTADOS E DISCUSSÕES}

\subsection{CONSULTA DE PREVENÇÃO DE INCAPACIDADES EM HANSENÍASE}


$\mathrm{O}$ atendimento e atuação da LADES ocorre durante as consultas de Enfermagem no referido ambulatório, em sala reservada, com material do próprio serviço, em companhia da enfermeira do setor. As consultas são norteadas por protocolos e manuais do Ministério da Saúde. Por turno, são atendidos entre 3 e 5 pacientes, conforme a demanda, as consultas duram em média de 30 a 45 minutos por paciente.

Através da comunicação terapêutica e da escuta ativa se estabelece o vínculo e a relação de confiança entre o profissional acadêmico e o paciente, em que este relata suas principais queixas e dúvidas sobre o tratamento e as peculiaridades da doença, refere sobre reações medicamentosas, adesão ao tratamento, e implicações da doença no seu convívio social e familiar. Em contrapartida, o cliente recebe apoio emocional, orientações e informações acerca da enfermidade, de forma a favorecer a compreensão e a aceitação da doença e do tratamento. O cliente recebe a Poliquimioterapia (PQT) de acordo com a classificação operacional da doença, fornecido pela farmácia do próprio hospital, e a dose mensal supervisionada do tratamento é administrada durante a consulta.

Realiza-se a avaliação neurológica simplificada no diagnóstico, mensalmente nos pacientes em segmento, e após a alta por cura contemplando: inspeção, percussão e palpação de nervos periféricos, avaliação de trofismo e força muscular pela técnica voluntary muscle test (VMT), avaliação da sensibilidade córnea pelo método de fio dental, avaliação de áreas hiposensitivas pelo exame com monofilamentos de SemmesWeinstein, em regiões palmar e plantar, e classificação do grau de incapacidade conforme preconizado pelo Ministério da Saúde (MS) e OMS (BRASIL, 2008). É feito o registro das consultas, das demandas encontradas e das condutas tomadas em prontuário e as alterações neurais encontradas são registradas no Formulário Para Avaliação Neurológica Simplificada do MS (BRASIL, 2008). Quando observada a necessidade, conforme demanda apresentada pelo paciente, ainda realizam-se encaminhamentos para consultas com outros profissionais especializados.

\subsection{ALTERAÇÕES FUNCIONAIS GERADAS PELA HANSENÍASE E ORIENTAÇÕES PARA O AUTOCUIDADO.}

Dentre as consultas realizadas foram observados os seguintes acometimentos nos pacientes: dores neuropáticas, espessamento de nervos periféricos, ressecamento e descamação 
da pele, ressecamento de nariz e olhos, alteração de sensibilidade palmar e plantar, diminuição da força muscular da mão, amiotrofia dos músculos da mão, mão em garra, rígida e móvel, e mal perfurante plantar ou úlcera neuropática causada por hanseníase.

Alguns pacientes já chegam para a primeira consulta com comprometimentos graves classificados como grau 2 de incapacidade, com o mal perfurante plantar, amiotrofia dos músculos da mão, e formação de mão em garra, o que sugere uma deficiência no diagnóstico precoce da doença.

Araújo, et al. (2014) em seu estudo que investigou as complicações neurais e o grau de incapacidades físicas nos olhos, mãos e pés antes e após o tratamento, também encontraram dados semelhantes, em que grande parte dos sujeitos com hanseníase foram diagnosticados tardiamente, já apresentando alterações neurais de alto poder incapacitante, de grau 1 e 2 no momento do diagnóstico.

Observou-se no histórico de alguns pacientes com comprometimento físicos instalados a demora do diagnóstico diferencial da hanseníase em suas fases iniciais, sendo realizados tratamento para outras doenças de sintomas parecidos com os da hanseníase durante anos.

As queixas de câimbras e formigamentos em mãos e pés, podem ser comuns, bem como artralgias com exames investigativos positivos para artrite reumatoide, o que pode atrasar o diagnóstico (Florêncio, 2018).

Observou-se ainda a demora em procurar o serviço de saúde por parte de alguns pacientes por desconhecimento dos sinais e sintomas da hanseníase, os quais realizavam automedicação para tratar as manchas hipocrômicas da hanseníase indeterminada com medicações para pitiríase versicolor ("pano branco").

Duas pacientes apresentaram queimaduras, uma de $2^{\circ}$ e outra de $3^{\circ}$ grau, localizadas em antebraço, por acidentes domésticos que as mesmas relataram não perceber nem sentir dor, desconhecendo o momento do acidente.

Para cada comprometimento identificado foram realizadas orientações de autocuidado baseadas em orientações do Ministério da Saúde na cartilha Autocuidado em hanseníase: face, mãos e pés, conforme disposto no Quadro 1. Também, realizou-se orientações sobre como realizar comunicação de contatos à unidade básica de saúde, e educação em saúde sobre a hanseníase de forma geral, seus possíveis agravantes e complicações, possíveis reações adversas ao tratamento, sensibilizando quanto à necessidade 
de continuidade e adesão ao tratamento. Além disso, procurou-se ouvir ativamente as ansiedades e os relatos sobre as situações vivenciadas pelos pacientes no contexto de suas relações sociais e familiares.

Araújo et al. (2016), em estudo que caracterizou a qualidade de vida de pessoas com hanseníase em tratamento ambulatorial, afirmam que aspectos físicos e emocionais são de fundamental importância na abordagem ao paciente com hanseníase, não devendo ser levado em conta apenas o processo de adoecimento físico.

Quadro 1 - Principais Comprometimentos Encontrados Durante Avaliação Neurológica Simplificada e Orientações de Autocuidado Correspondente. Fortaleza, 2018.

\begin{tabular}{|c|c|}
\hline COMPROMETIMENTOS & $\begin{array}{c}\text { ORIENTAÇÕES DE AUTOCUIDADO } \\
\text { CORRESPONDENTE }\end{array}$ \\
\hline Ressecamento dos olhos & $\begin{array}{l}\text { Usar o colírio lubrificante ocular conforme prescrito; } \\
\text { realizar exercícios de piscar várias vezes ao dia; fazer } \\
\text { autoavaliação no espelho pelo menos uma vez ao dia } \\
\text { atentando-se para qualquer alteração. }\end{array}$ \\
\hline Ressecamento do nariz & $\begin{array}{l}\text { Realizar lavagem nasal de } 3 \text { a } 4 \text { vezes ao dia com água } \\
\text { limpa aspirando de vagar; manter autoavaliação no } \\
\text { espelho diariamente em busca de feridas ou } \\
\text { sangramento. }\end{array}$ \\
\hline Ressecamento e descamação da pele & $\begin{array}{l}\text { Utilizar bacia ou balde com água limpa da torneira em } \\
\text { temperatura ambiente para deixar mãos e braços } \\
\text { submersos por } 10 \text { minutos, em seguida sacudir para } \\
\text { retirar o excesso, e com mãos e braços ainda úmidos } \\
\text { passar hidrante corporal ou óleo mineral; realizar os } \\
\text { mesmos procedimentos em pernas e pés pelo menos } 2 \\
\text { vezes ao dia. }\end{array}$ \\
\hline Alteração de sensibilidade palmar e plantar & $\begin{array}{l}\text { Sempre examinar mãos e pés em busca de vermelhidão, } \\
\text { ferimentos, inchaços ou calos; proteger mãos e pés em } \\
\text { qualquer atividade domésticas, de laser, ou trabalho, } \\
\text { com luvas ou calçados confortáveis; proteger-se de } \\
\text { queimaduras adaptando, com borracha ou pano grosso, } \\
\text { cabos de panelas, xícaras, talheres e pratos; manter } \\
\text { cuidado redobrado ao manipular alimentos quentes ou } \\
\text { próximo ao fogo; sempre procurar ajuda do serviço de } \\
\text { saúde em acaso de ferimentos ou acidentes domésticos. }\end{array}$ \\
\hline
\end{tabular}

continua 
Quadro 2 - Principais Comprometimentos Encontrados Durante Avaliação Neurológica Simplificada e Orientações de Autocuidado Correspondente. Fortaleza, 2018.

continuação

Realizar exercícios de alongamento para mãos e dedos ao menos 3 vezes ao dia em seções de 10 movimentos; em posição sentada colocar dorso do braço e mãos sobre a perna, em seguida com uma das mãos esticar a

Diminuição da força muscular da mão, amiotrofia dos músculos da mão, e ou mão em garra. outra passando a mão sobre antebraço até os dedos; levantar os dedos sem ajuda; separar os dedos contra resistência usando liga de elástico; esticar o polegar com ajuda da outra mão; abrir o polegar sem ajuda e contra a resistência; fazer exercício de pinça com pegador; erguer punho segurando objeto leve, bola de borracha ou lata de refrigerante.

Manter os pés em repouso sempre que possível; evitar pisar com o pé ferido; usar calçados e palmilhas adequados que ofereçam conforto e proteção aos pés; examinar sempre os pés com ajuda de um espelho no chão para facilitar visualização; buscar acompanhamento na unidade básica de saúde para cuidados e troca de curativos.

Fonte: Autores, baseado nas orientações da cartilha de autocuidado em hanseníase do MS (2010).

\section{CONCLUSÃO}

As atividades de extensão realizadas no ambulatório de dermatologia constituem um meio propício de aprendizado no campo da hanseníase uma vez que proporcionam: autonomia ao acadêmico no desenvolvimento da consulta de Enfermagem, contato prático com as diversas apresentações da doença preparando para o reconhecimento de sinais e sintomas, recidivas, reações à medicação quimioterápica e tratamento alternativo, desenvoltura e manejo no trato com usuários do serviço público, e compartilhamento científico das atividades assistenciais desenvolvidas de forma gratuita para esse público.

Além disso, a avaliação neurológica simplificada, as orientações de autocuidado e a criação de vínculo estabelecidas no seguimento dos pacientes, monitoram o estado em que estes se encontram e contribuem para identificação precoce de complicações neurais prevenindo incapacidades, como afirmam diversos estudiosos. 


\section{REFERÊNCIAS}

ARAÚJO, A. R. et al. Complicações neurais e incapacidades em hanseníase em capital do nordeste brasileiro com alta endemicidade. Revista Brasileira de Epidemiologia, v. 17, n. 4, p. 899-910, 2014. Disponível em: <http://www.scielo.br/pdf/rbepid/v17n4/pt_1415-790Xrbepid-17-04-00899.pdf >. Acesso em: 31 de agosto de 2018.

ARAÚJO, D.A.L. et al. Caracterização da qualidade de vida de pessoas com hanseníase em tratamento ambulatorial. Revista de Pesquisa: Cuidado é Fundamental Online, [S.I.], v. 8, n. 4, p. 5010-5016, out. 2016. ISSN 2175-5361. Disponível em:

<http://www.seer.unirio.br/index.php/cuidadofundamental/article/view/4732>. Acesso em: 31 de agosto de 2018.

ALVES, C.J.M. et al. Avaliação do grau de incapacidade dos pacientes com diagnóstico de hanseníase em serviço de dermatologia do estado de São Paulo. Revista da Sociedade Brasileira de Medicina Tropical, Uberaba, v. 43, n. 4, p. 460-461, ago. 2010. Disponível em: <http://www.scielo.br/scielo.php?script=sci_arttext\&pid=S0037-

86822010000400025\&lng=en\&nrm=iso>. Acesso em: 01 de setembro de 2018.

BRASIL. Guia prático sobre a hanseníase. Secretaria de Vigilância em Saúde. Departamento de Vigilância e Doenças Transmissíveis - Brasília: Ministério da Saúde: 35 p. 2017.

Autocuidado em hanseníase: face, mãos e pés. Secretaria de Vigilância Epidemiológica. Departamento de Vigilância Epidemiológica. 1. ed. Ministério da Saúde: Brasília, 2010.

Manual de Prevenção de Incapacidades. Cadernos de prevenção e reabilitação em hanseníase; n. 1. Secretaria de Vigilância em Saúde. Departamento de Vigilância Epidemiológica. ed. Ministério da Saúde: Brasília, 2008.

CEARÁ. Boletim Epidemiológico Hanseníase. Secretaria da Saúde do Estado do Ceará. Fortaleza 2017. p. 2.

DUARTE, M.T.C. et al. Consulta de enfermagem: estratégia de cuidado ao portador de hanseníase em atenção primária. Texto Contexto Enfermagem, Florianópolis, v. 18, n. 1, p. 100-7, Jan-Mar 2009. Disponível em:

<http://www.scielo.br/pdf/tce/v18n1/v18n1a12>. Acesso em: 29 de agosto de 2018.

FLORÊNCIO, C.M.G.D. Docentes da UFC: Padrões espaciais, temporais e clínicoepidemiológicos da hanseníase no Brasil, 2001 a 2015. Tese (doutorado) - Universidade Federal do Ceará, Faculdade de Medicina, Programa de Pós-Graduação em Saúde Coletiva, Fortaleza, 2018.

ORGANIZAÇÃO MUNDIAL DA SAÚDE. Estratégia global para hanseníase (2016-2020). Aceleração rumo a um mundo sem hanseníase. New Delhi, India, 2015. Disponível em: < http://apps.who.int/iris/bitstream/handle/10665/208824/9789290225201pt.pdf;jsessionid=131 0D0D157FF66396335F9E6FE64124E?sequence=17>. Acesso em: 23 ago. 2018. 